\title{
Reduced Oxidative Activities in Escherichia coli and Bacillus megaterium in Relation to Other Changes during Inhibition of Growth by Streptomycin
}

\author{
By R. HANCOCK \\ Department of Bacteriology, University of Manchester
}

(Received 20 January 1961)

\begin{abstract}
SUMMARY
When the growth of cultures of Bacillus megaterium KM, Escherichia coli B or Bacillus subtilis ceased following addition of streptomycin during the exponential phase of growth, harvested organisms showed oxidative activities on glucose, lactate, pyruvate and succinate which were between 20 and $60 \%$ of those of normal organisms. With $E$. coli, this reduction in oxidative activity was closely parallel to the decrease in growth rate. During the period in which growth became fully inhibited by streptomycin, the total quantities of amino acids and $260 \mathrm{~m} \mu$ absorbing compounds in hot water extracts of $\boldsymbol{E}$. coli and $\boldsymbol{B}$. megaterium did not differ significantly from those in normal organisms. Protoplasts could be prepared in normal yield from streptomycin-inhibited organisms of both these species; such protoplasts showed normal stability and had internal osmotic pressures which differed only slightly from normal. From these results it appears unlikely that there was any general breakdown of the permeability barrier of these organisms during inhibition of growth by streptomycin. Lysates of protoplasts from streptomycininhibited Bacillus megaterium showed oxidative activities which were also decreased to between $20 \%$ and $45 \%$ of those of lysates from normal organisms. With succinate and malate as substrates this difference appeared to be due predominantly to a considerable decrease in the activity of the sedimentable 'ghost' fraction of the lysed protoplasts.
\end{abstract}

\section{INTRODUCTION}

During the period in which growth of cultures of Staphylococcus aureus strain Duncan becomes inhibited by streptomycin, there is a progressive decrease in the oxidative activities of harvested organisms which parallels the decrease in the rates of synthesis of cell constituents (Hancock, 1960a). In organisms harvested from streptomycin-inhibited cultures, the $Q_{\mathrm{O}_{2}}$ values for oxidation of all substrates are diminished to between 5 and $\mathbf{5 0} \%$ of the values for normal organisms. Similar observations were made by Jackson (1958) with another strain of $S$. aureus. In $S$. aureus strain Duncan this change was the major alteration observed in biochemical activities of streptomycin-inhibited organisms, and we therefore looked for similar changes in other organisms during inhibition of growth by streptomycin. Attempts have also been made to relate the observed changes in oxidative activity with any general alteration in the permeability of the cells. In Escherichia coli strain W, changes occur in the permeability of the cell membrane during inhibition of growth 
by streptomycin (Anand \& Davis, 1960), and there is evidence that the membrane is the site of an early, but not the bactericidal, action of streptomycin (Drs P. Plotz \& B. D. Davis, personal communication). We have also investigated oxidative activities in some subcellular fractions of Bacillus megaterium after growth in the presence of streptomycin. Some of these results have been published in abstract form (Hancock, 1960b).

\section{METHODS}

Organisms, growth and harvesting. Escherichia coli strain B was grown in C medium (McQuillen \& Roberts, 1954) containing glucose (1 mg./ml.) and adjusted to $\mathrm{pH} \mathbf{7 \cdot 2}$; Bacillus megaterium strain KM and a laboratory strain of Bacillus subtilis were grown in nutrient broth (Oxoid no. 2) at $\mathrm{pH}$ 7.5. All cultures were grown in flasks shaken at $37^{\circ}$ in a constant temperature water bath; up to $100 \mathrm{ml}$. of medium was used in $250 \mathrm{ml}$. Erlenmeyer flasks fitted with side arms to fit the Unicam SP. 600 spectrophotometer; larger cultures were grown in 2 l. flasks containing $750 \mathrm{ml}$. of medium. The pre-warmed growth medium was inoculated with $10 \%(\mathrm{v} / \mathrm{v})$ of an overnight culture grown in the same medium in $150 \mathrm{ml}$. tubes through which a stream of air was bubbled.

Growth was followed by determining the optical density of samples of a culture at $700 \mathrm{~m} \mu$ by using the Unicam SP. 600 spectrophotometer. When required, streptomycin was added to a portion of a culture during the exponential phase of growth; the remainder of the culture then served as a control.

Organisms were harvested from cultures of small volume (100 ml. or less) on membrane filters as described previously (Hancock, 1960 a); larger cultures were harvested by centrifugation at $6000 \mathrm{~g}$ for $5 \mathrm{~min}$. at room temperature. Escherichia coli was washed by resuspension in ice-cold $0.5 \mathrm{M}-\mathrm{NaCl}$, since distilled water removes some low-molecular weight compounds from this organism (Britten, 1956). For manometric experiments, Bacillus megaterium and $B$. subtilis were washed in distilled water; however, such washing was found to decrease the yield and stability of protoplasts from B. megaterium and was omitted when these were to be prepared, since the protoplasts were effectively washed by centrifugation from the 'protoplasting' medium before use.

Manometric techniques. For intact organisms, the conditions were those previously used for Staphylococcus aureus (Hancock, 1960 a). For determining oxidative activity in protoplast lysates and subcellular fractions of Bacillus megaterium, 'lysing medium' (see below) was used in manometer flasks in place of the phosphate buffer used for intact organisms. All determinations were carried out in duplicate.

Hot water extracts of organisms were prepared as described previously (Hancock, $1960 a$ ) after harvesting on membrane filters and resuspension.

Preparation and fractionation of protoplasts. Protoplasts of Bacillus megaterium were prepared as described by Weibull, Beckman \& Bergström (1959) with sucrose as osmotic stabilizer, but using an initial suspension concentration equivalent to about $15 \mathrm{mg}$. dry wt. of organisms $/ \mathrm{ml}$. When protoplasts were to be prepared simultaneously from normal organisms and streptomycin-inhibited organisms, the volumes of the two suspensions were adjusted initially to give an equal dry weight of organisms $/ \mathrm{ml}$. in each. In all subsequent stages the volumes were also adjusted so that the final concentration of protein in the total lysates and other fractions 
were similar in the two preparations. Centrifugations were carried out in the No. 40 rotor of the Spinco model L ultra-centrifuge, with refrigeration. Protoplast formation was followed with the phase-contrast microscope, and was complete in 15-20 min. at room temperature. The protoplasts were centrifuged (4000 $\mathrm{g}, 10 \mathrm{~min}$.), and lysed by suspension in ice-cold 0.04 M-potassium phosphate buffer ( $\mathrm{pH} \mathrm{7 \cdot 0}$ ) containing 0.002 M- $\mathrm{MgSO}_{4}$ ('lysing medium'; Storck \& Wachsman, 1957); crystalline deoxyribonuclease (L. Light and Co., Ltd., Colnbrook, Bucks) was added to a final concentration of $1 \mu \mathrm{g} . / \mathrm{ml}$. When the viscosity of the lysates had decreased to a normal level, they were used immediately for manometry or further fractionation. The 'ghost' fraction was separated from such lysates by centrifugation at $25,000 \mathrm{~g}$ for $15 \mathrm{~min}$., washed once by a similar centrifugation from lysing medium, and resuspended in a volume of cold lysing medium approximately equal to that of the original total lysate.

For manometric experiments particular care was taken that protoplast formation was complete before harvesting and lysis, and that no intact organisms remained in the lysate; on the very infrequent occasions when intact organisms were detected after a thorough microscopic examination, the preparation was discarded. Manometer flasks contained a volume of total lysate containing 5-10 mg. protein, of 'ghost' fraction containing $2-3 \mathrm{mg}$. protein, or of supernatant fraction containing 5-8 mg. protein.

Osmotically sensitive forms of Escherichia coli were formed by the procedure of Mahler \& Fraser (1956) using lysozyme and ethylenediaminetetra-acetic acid in $0.5 \mathrm{M}$-sucrose, and a suspension density of 10-15 mg. dry weight of organisms $/ \mathrm{ml}$. Such forms will be referred to here as protoplasts for the sake of convenience only, bearing in mind the reservations of Brenner et al. (1958). The development of osmotic sensitivity in suspensions of $E$. coli was followed by pipetting duplicate samples $(0.1 \mathrm{ml}$.) of each suspension into two $2.5 \mathrm{ml}$. samples of $0.01 \mathrm{M}$-potassium phosphate buffer ( $\mathrm{pH} \mathrm{6.8)}$ ) one of which contained $0.5 \mathrm{M}-\mathrm{NaCl}$; the ratio of the optical density $(700 \mathrm{~m} \mu)$ in buffer to that in buffer $+\mathrm{NaCl}$ decreased as fragility developed, and after about 15-20 min. reached a minimum value of $0 \cdot 18-0 \cdot 20$.

The yield of protoplasts from suspensions of both Escherichia coli and Bacillus megaterium was estimated by comparing the initial optical density of the suspension with the final optical density when protoplast formation was complete; for this purpose a lower suspension density, 2-3 mg. dry weight of organisms/ml., was used.

Determination of internal osmotic pressure of protoplasts. The osmotic pressure within protoplasts was estimated by a procedure based on method III of Mitchell \& Moyle (1956 a); this depends on determination of the concentration of external solute which is necessary to protect protoplasts against osmotic lysis. Samples (0.1 ml.) of a suspension of protoplasts were pipetted into $2.5 \mathrm{ml}$. samples of $0.01 \mathrm{M}$-potassium phosphate buffer ( $\mathrm{pH} \mathrm{6.8)}$ containing $\mathrm{NaCl}$ at concentrations from 0 to $0.5 \mathrm{M}$. After 20 min., lysis in the more dilute solutions was complete and the optical density (at $700 \mathrm{~m} \mu$ ) of each sample was determined.

Streptomycin. The preparations used in these experiments, and the preparation of solutions, were as described previously (Hancock, 1960a).

Amino acids and dry weights of bacterial suspensions were determined as described previously (Hancock, $1960 \mathrm{a}$ ). Substances absorbing light at $260 \mathrm{~m} \mu$ were estimated by using the Unicam SP. 700 spectrophotometer. 
Protein was estimated by the method of Lowry, Rosebrough, Farr \& Randall (1951) using suitably diluted samples of the preparations; crystalline lysozyme was used as a standard.

\section{RESULTS}

Diminished oxidative activity in streptomycin-inhibited organisms

Streptomycin was used at concentrations which resulted in complete inhibition of growth of the organisms after about $60 \mathrm{~min}$., so that the relative time of occurrence of other changes during this period could be determined. In initial experiments
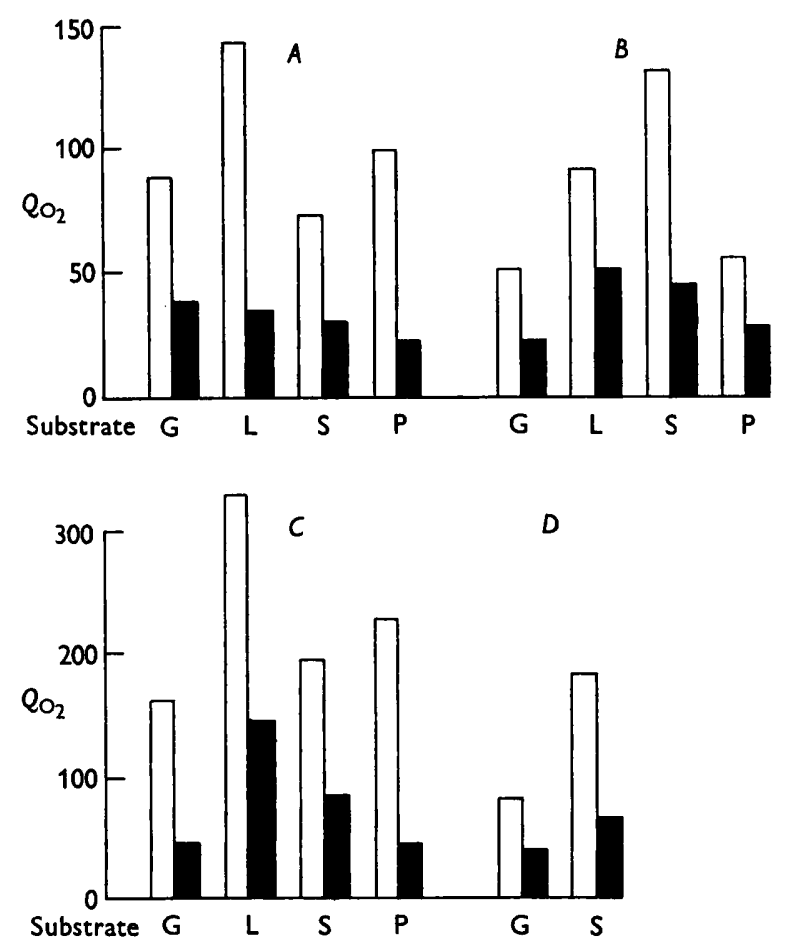

Fig. 1. $Q_{\mathrm{O}_{2}}$ values of organisms harvested simultaneously from control cultures (open columns) and streptomycin-inhibited cultures (solid columns). $A$ and $B$. Bacillus megaterium KM; streptomycin sulphate $2 \mu \mathrm{g} . / \mathrm{ml}$. $A$, harvested after $15 \mathrm{~min}$. , growth inhibition just detectable; $B$, harvested after 75 min., growth just ceased. (Two separate experiments.) $C$, Escherichia coli B, streptomycin sulphate $40 \mu \mathrm{g} . / \mathrm{ml}$. harvested after $40 \mathrm{~min}$., growth just ceased. $D$, Bacillus subtilis, streptomycin sulphate $150 \mu \mathrm{g} . / \mathrm{ml}$. harvested after 60 min., growth just ceased. Substrates: $\mathbf{G}=$ glucose; $\mathbf{L}=$ lactate; $\mathbf{S}=$ succinate; $\mathbf{P}=$ pyruvate.

using Escherichia coli, Bacillus megaterium and B. subtilis, the organisms were harvested immediately after growth had finally ceased in the presence of streptomycin, and their ability to oxidize a number of substrates was compared with that of organisms harvested from a parallel control culture at the same time. The results of these experiments are shown in Fig. 1; $Q_{\mathrm{O}_{2}}$ values were also determined for Bacillus megaterium with organisms harvested when the inhibition of growth by streptomycin was first detectable. When inhibition of growth was complete, the 
oxidative activities of harvested organisms were decreased in all cases to values considerably below those of normal organisms; with $B$. megaterium, some decrease had already occurred when growth inhibition was first detectable.

To relate these changes more closely to the inhibition of growth, the progress of the decrease of $Q_{\mathrm{O}_{2}}$ (glucose) was compared with the development of growth inhibition in cultures of Escherichia coli, with the results shown in Fig. 2. With $E$. coli the $Q_{\mathrm{o}_{2}}$ (glucose) and the growth rate began to decrease at about the same time, and the $Q_{\mathrm{o}_{2}}$ had reached about $30 \%$ of the control value when growth inhibition was complete. These observations are in general very similar to those made with Staphylococcus aureus (Hancock, 1960a).

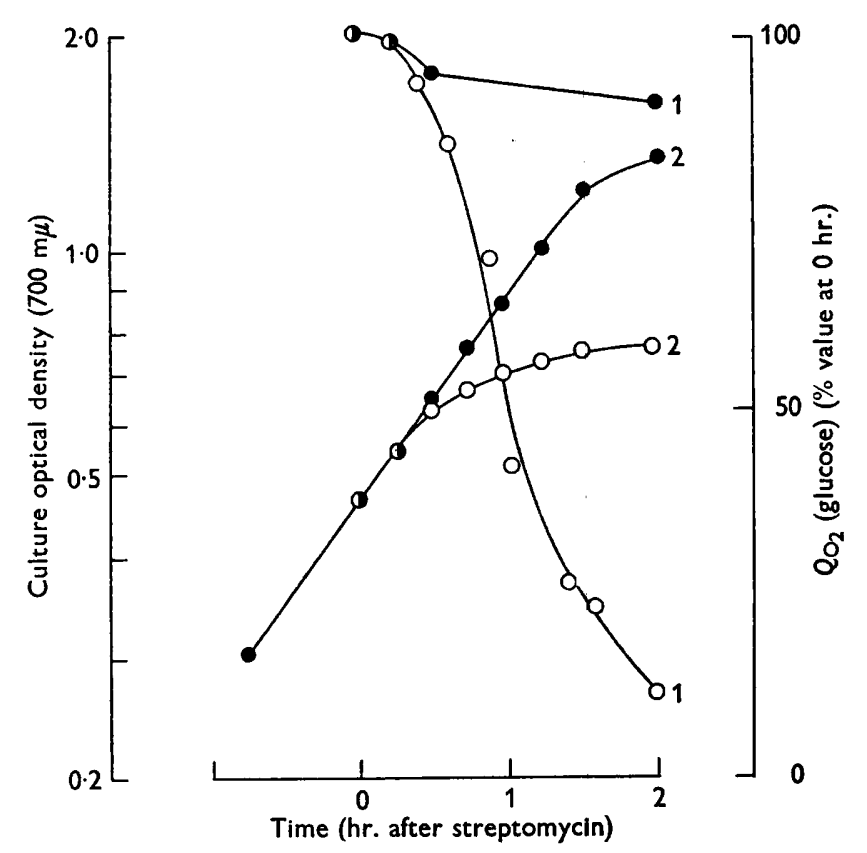

Fig. 2. $Q_{\mathrm{O}_{2}}$ (glucose) values (curves 1 ) of Escherichia coli $\mathrm{B}$ harvested from control cultures $(0)$ and cultures after addition of streptomycin sulphate $60 \mu \mathrm{g} . / \mathrm{ml}$. (O). Curves 2 represent optical densities of the cultures.

Anand \& Davis (1960) showed that during the bactericidal action of streptomycin on Escherichia coli W, changes occurred in the permeability of the cells which result in the excretion of some intracellular compounds. Changes in permeability could clearly lead to loss of oxidative activity because of loss of respiratory coenzymes, and it was important to determine whether, in the organisms investigated here, any changes in the permeability barrier could be observed during the period in which oxidative activity decreased. No significant decrease in the total quantity of amino acids, $260 \mathrm{~m} \mu$-absorbing substances or phosphate compounds was earlier observed with Staphylococcus aureus during inhibition of growth (Hancock, 1960a). 


\section{Hot-water extractable compounds during inhibition of growth}

In the experiment with Escherichia coli illustrated in Fig. 2, samples of the suspension of organisms whose $Q_{\mathrm{O}_{2}}$ values were to be determined were taken for estimation of the total quantities of amino acids and $260 \mathrm{~m} \mu$-absorbing compounds in hot-water extracts of the organisms. No large differences were found between the quantities of these compounds in normal organisms and in organisms harvested during the period in which growth became inhibited and in which the $\boldsymbol{Q}_{\mathrm{o}_{2}}$ (glucose) decreased to $30 \%$ or less of the normal value (Table 1 ). The quantity of amino acids did however begin to decrease after inhibition of growth had become complete. Table 1 includes data obtained in similar experiments with Bacillus megaterium. From these results, it appears that the permeability barrier of $\boldsymbol{E}$. coli and $\boldsymbol{B}$. megaterium remained intact, at least to the extent that there was no general leakage of intracellular compounds of low molecular weight from the cells during the period in which growth was inhibited.

Table 1. Quantities of some compounds in hot-water extracts of Bacillus megaterium $K M$ and Escherichia coli $B$ during inhibition of growth by streptomycin

The estimations for $\boldsymbol{E}$. coli $\mathbf{B}$ were made on the same suspensions as those used in the experiment in Fig. 2. For B. megaterium, the streptomycin sulphate concentration was $2 \mu \mathrm{g} . / \mathrm{ml}$.; inhibition of growth was detectable at $45 \mathrm{~min}$. and complete by $75 \mathrm{~min}$.

\begin{tabular}{|c|c|c|c|c|c|}
\hline \multirow[b]{2}{*}{ Organism } & \multirow{2}{*}{$\begin{array}{c}\text { Time after } \\
\text { streptomycin } \\
\text { added (min.) }\end{array}$} & \multicolumn{2}{|c|}{$\begin{array}{l}\mu \text { mole amino } \\
\text { acids/g. } \\
\text { dry weight } \\
\text { organisms }\end{array}$} & \multicolumn{2}{|c|}{$\begin{array}{l}\text { Optical density } \\
(260 \mathrm{~m} \mu) / \mathrm{g} . \\
\text { dry weight } \\
\text { organisms }\end{array}$} \\
\hline & & $\mathbf{C}$ & $\mathbf{S}$ & $\mathbf{C}$ & $\mathbf{S}$ \\
\hline \multirow[t]{5}{*}{ E. coli B } & $\mathbf{0}$ & 105 & 105 & 746 & 746 \\
\hline & 30 & - & 94 & - & 1020 \\
\hline & 60 & 96 & 88 & 817 & 900 \\
\hline & 90 & 99 & 102 & 746 & 904 \\
\hline & 120 & 99 & 51 & 902 & 821 \\
\hline \multirow[t]{3}{*}{ 8. megaterium $\mathbf{K M}$} & $\mathbf{0}$ & - & - & 1020 & 1020 \\
\hline & 45 & 111 & 110 & 1000 & 1005 \\
\hline & 75 & 159 & 145 & 800 & 952 \\
\hline
\end{tabular}

Production and stability of protoplasts from streptomycin-inhibited organisms

A second procedure used to investigate the integrity of the permeability barrier depends on comparison of the yield and stability of protoplasts from streptomycininhibited organisms and normal organisms. Protoplasts are stable only when suspended in solutions of non-penetrating solutes at concentrations which exert an osmotic pressure on the permeability barrier equal and opposite to that due to the intracellular solutes (for general discussion, see Mitchell \& Moyle, 1956b). If, in streptomycin-inhibited organisms, alterations were to occur in the permeability barrier which resulted in loss of its ability to exclude the stabilizing solute used (or other molecules such as streptomycin, for example), protoplasts from such organisms would not be stable in the presence of solutes which stabilize protoplasts from normal organisms. In the experiments described here, sucrose or $\mathrm{NaCl}$ were used 
as stabilizing solutes, and the optical density of a protoplast suspension was used to estimate the proportion of protoplasts which remained intact, as in the experiments of Mitchell \& Moyle $(1956 a, 1957)$. In all cases, streptomycin-inhibited organisms were harvested when inhibition of growth had just become complete; normal organisms were harvested simultaneously from a control culture. Figure 3 shows progress curves of the optical density of suspensions of normal and streptomycininhibited Bacillus megaterium and Escherichia cali, when protoplast formation occurred in the presence of sucrose at a concentration which conferred complete stability on protoplasts from normal organisms, and also when no stabilizer was present. In B. megaterium (Fig. $3 \mathrm{~A}$ ) the final yield of protoplasts from streptomycininhibited organisms in this experiment was about $20 \%$ lower than that from normal organisms; smaller differences in protoplast yield were obtained in subsequent experiments (Fig. $4 \mathrm{~A}$ ). In $\boldsymbol{E}$. coli (Fig. $\mathbf{3 B}$ ) there was only a very small difference in the final yield of protoplasts. With both organisms, protoplasts from streptomycin-inhibited organisms were stable for considerable periods of time, so that there appeared to be no leakage of the stabilizing solute into the protoplasts.
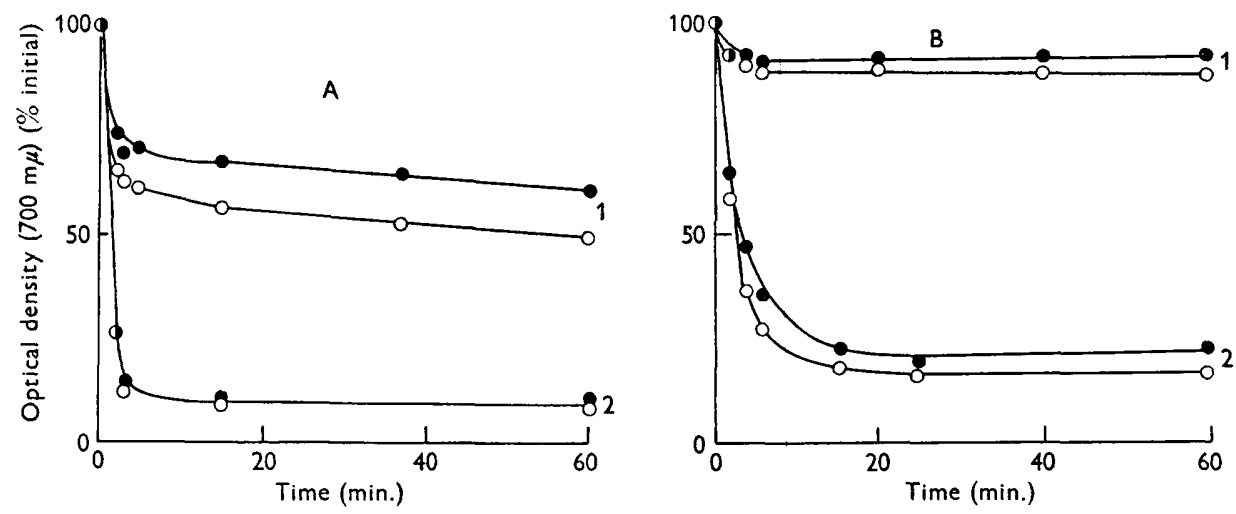

Fig. 3. Optical density of suspensions of Bacillus megaterium KM (A) and Escherichia coli $\mathbf{B}(\mathrm{B})$ during protoplast formation in the presence (curves 1 ) or absence (curves 2) of $0.5 \mathrm{M}$-sucrose. Bacteria from a culture containing streptomycin sulphate $(2 \mu \mathrm{g} . / \mathrm{ml}$. for $B$. megaterium, $50 \mu \mathrm{g} . / \mathrm{ml}$. for $E$. coli) were harvested when growth had just ceased (O), and control bacteria were harvested simultaneously (O).

The internal osmotic pressure of protoplasts was estimated by the procedure used by Mitchell \& Moyle $(1956 a, 1957)$ in which the proportion of intact protoplasts is determined over a range of external solute concentrations; the mean internal osmotic pressure is regarded as equal to that of the solution in which $50 \%$ of the protoplasts remain intact. This procedure gives values for the internal osmotic pressure which agree well with those obtained by more direct methods (Mitchell \& Moyle, $1956 b$ ). Figure 4 shows the relation between the concentration of $\mathrm{NaCl}$ in the suspending medium and the stability of protoplasts from normal and streptomycininhibited Bacillus megaterium and Escherichia coli. Very similar results were obtained when the stability was measured in solutions containing sucrose in place of $\mathrm{NaCl}$. The yield of protoplasts from the streptomycin-inhibited organisms, expressed relative to that from normal organisms, was $94 \%$ for $\boldsymbol{B}$. megaterium and $97 \%$ for $E$. coli. With $E$. coli protoplasts from streptomycin-inhibited organisms appear to 
have about the same mean internal osmotic pressure as those of normal organisms; with $B$. megaterium they had a mean internal osmotic pressure slightly higher than normal. The $Q_{\mathrm{O}_{2}}$ of the organisms used in this experiment were also determined at the same time; with $B$. megaterium the streptomycin-inhibited organisms had $Q_{\mathrm{O}_{2}}$ values between $34 \%$ (succinate) and $\mathbf{5 0} \%$ (lactate) of the control; with $\boldsymbol{E}$. coli between $28 \%$ (pyruvate) and $49 \%$ (succinate) of the control. From these experiments also it appears that no large leakage of intracellular solutes could have occurred with the streptomycin-inhibited organisms, since such a loss would have resulted in the possession, by protoplasts from these organisms, of a lower mean internal osmotic pressure than protoplasts from normal organisms. It was also intended to investigate the stability and internal osmotic pressure of 'protoplasts' of Staphylococcus aureus prepared by the procedure of Mitchell \& Moyle (1957);

A

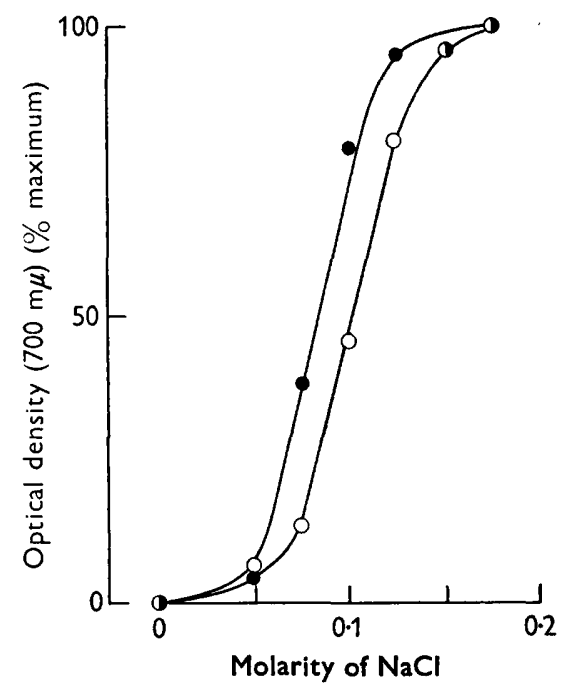

B

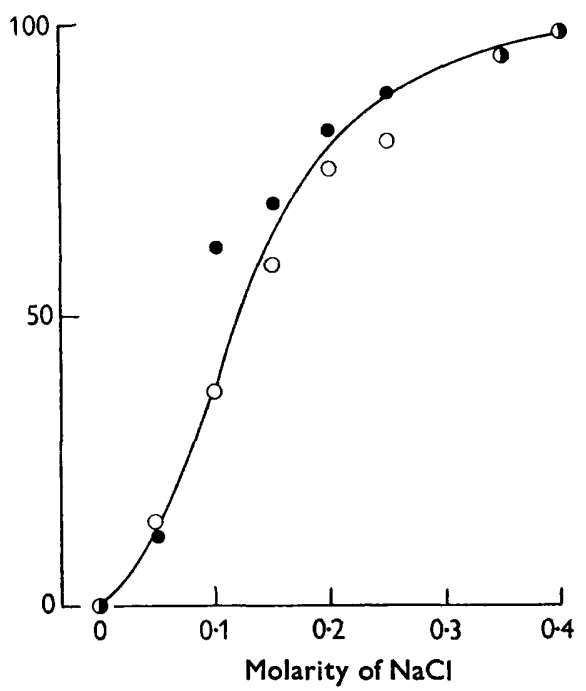

Fig. 4. Final optical densities of suspensions of protoplasts of Bacillus megaterium KM (A) and Escherichia coli $\mathrm{B}$ (B) after transfer to solutions containing $\mathrm{NaCl}$ at different molarities, expressed relative to the optical density in $0.5 \mathrm{M}-\mathrm{NaCl}$ in which all protoplasts were completely stable. Protoplasts prepared from normal cells $(\bullet)$ and streptomycininhibited cells (O), grown as in Fig. $\mathbf{3}$.

however, for reasons which are not apparent, no 'protoplast' formation could be obtained in suspensions of streptomycin-inhibited organisms, although normal organisms became osmotically fragile.

\section{Oxidative activities in lysed protoplasts of Bacillus megaterium}

Attempts were made to investigate oxidative activities in subcellular fractions from streptomycin-inhibited cells of Bacillus megaterium. The distribution of a number of oxidative systems in cells of this organism was investigated by Storck \& Wachsman (1957) who used a procedure in which the organisms were first converted to protoplasts, which were then lysed osmotically. The total lysates were further separated into a sedimentable fraction containing 'ghosts' (probably protoplast membranes) and a supernatant fraction. A similar procedure was followed here. 
From each suspension of organisms which were to be converted to protoplasts, a sample was first taken to determine the $Q_{\mathrm{o}_{2}}$ values of the intact organisms for subsequent comparison with the activities of subcellular preparations.

\section{Table 2. Oxidative activities in intact cells and total lysates of Bacillus megaterium $K \boldsymbol{M}$}

\begin{tabular}{|c|c|c|c|c|c|}
\hline \multicolumn{6}{|l|}{ Organisms 1} \\
\hline \multirow[b]{2}{*}{$\begin{array}{c}\text { Pre- } \\
\text { paration }\end{array}$} & \multirow[b]{2}{*}{ Substrate } & \multicolumn{2}{|c|}{$\begin{array}{c}Q_{\mathrm{O}_{2}} \\
\text { (Intact organisms) }\end{array}$} & \multicolumn{2}{|c|}{$\begin{array}{l}\mu \mathrm{l} . \mathrm{O}_{2} / \mathrm{mg} \text {. protein } / \mathrm{hr} \text {. } \\
\text { (Total lysate) }\end{array}$} \\
\hline & & Control & $\begin{array}{c}\text { Strepto- } \\
\text { mycin- } \\
\text { inhibited }\end{array}$ & Control & $\begin{array}{c}\text { Strepto- } \\
\text { mycin- } \\
\text { inhibited }\end{array}$ \\
\hline 1 & $\begin{array}{l}\text { Glucose } \\
\text { Succinate } \\
\text { Lactate }\end{array}$ & $\begin{array}{l}187 \\
148 \\
161\end{array}$ & $\begin{array}{l}45 \cdot 2 \\
24 \cdot 9 \\
18 \cdot 1\end{array}$ & $\begin{array}{l}13 \cdot 7 \\
40 \cdot 1 \\
24 \cdot 1\end{array}$ & $\begin{array}{r}3 \cdot 5 \\
11 \cdot 6 \\
11 \cdot 4\end{array}$ \\
\hline 2 & $\begin{array}{l}\text { Succinate } \\
\text { Lactate }\end{array}$ & $\begin{array}{l}192 \\
148\end{array}$ & $\begin{array}{l}32 \cdot 0 \\
25 \cdot 2\end{array}$ & $\begin{array}{l}31 \cdot 4 \\
61 \cdot 4\end{array}$ & $\begin{array}{l}14 \cdot 0 \\
33 \cdot 6\end{array}$ \\
\hline 3 & Succinate & 242 & $24 \cdot 2$ & 46.5 & $10 \cdot 8$ \\
\hline 4 & Succinate & 225 & $20 \cdot 8$ & $32 \cdot 8$ & $14 \cdot 6$ \\
\hline
\end{tabular}

Table 2 shows the rate of oxidation of some substrates in total lysates of protoplasts from a number of batches of normal and streptomycin-inhibited Bacillus megaterium. In each experiment the streptomycin-inhibited organisms were harvested when growth had just ceased, and a control culture was harvested simultaneously. The proportion of the oxidative activity of the intact organisms which was recovered in total lysates ranged between about $3 \%$ for glucose (preparation 1) to $20 \%$ for lactate (preparation 2 ). The recoveries of activity for succinate and lactate were somewhat higher than those obtained by Storck \& Wachsam (1957). In all the preparations, lysates from streptomycin-inhibited $B$. megaterium showed a smaller activity, relative to their protein content, than those from normal organisms. The difference in activity in the total lysates was in most cases somewhat lower than that in the corresponding intact organisms. The addition of streptomycin (up to $100 \mu \mathrm{g} . / \mathrm{ml}$.) to the contents of the manometer flasks had no effect on the oxidative activities of preparations from normal organisms.

\section{Oxidative activities in fractionated lysates}

The systems responsible for the oxidation of succinate and malate were chosen for further investigations; the total activity of these systems is divided about equally between the 'ghost' and supernatant fractions, but the specific activity of the 'ghost' fraction is 3 to 6 times that of the supernatant (Storck \& Wachsman, 1957). It was also found here that activities on these two substrates were more consistent and reproducible than those obtained with other substrates. Although Storck \& Wachsman (1957) found that certain co-factors stimulated oxidative activities in their preparations, the addition of adenosine diphosphate, di- or triphosphopyridine nucleotide, or cytochrome $c$, either singly or together, resulted in no stimulation of the preparations used here.

The distribution of oxidative activities in these fractions from two batches of 
normal and streptomycin-inhibited Bacillus megaterium are summarized in Table 3. In various preparations, the 'ghost' fraction contained between 15 and $20 \%$ of the protein content of the total lysate, similar to the value found by Storck \& Wachsman (1957). These workers observed that the sum of malate oxidizing activity in the two fractions was greater than that in the original total lysate, as was also observed here.

Table 3. Oxidative activity in fractionated total lysates of Bacillus megaterium $K M$

Conditions of growth as in Table 2; $\mathrm{C}=$ control organisms, $\mathbf{S}=$ streptomycin-inhibited organisms

\begin{tabular}{|c|c|c|c|c|c|c|}
\hline \multirow[b]{2}{*}{ Preparation } & \multirow[b]{2}{*}{ Substrate } & \multirow[b]{2}{*}{ Fraction } & \multicolumn{2}{|c|}{$\begin{array}{c}\text { Specific activity } \\
\mu \mathrm{l} . \mathrm{O}_{2} / \mathrm{mg} \text {. protein } / \mathrm{hr} \text {. }\end{array}$} & \multicolumn{2}{|c|}{$\%$ activity of total lysat } \\
\hline & & & C & $\mathbf{s}$ & C & $\mathbf{S}$ \\
\hline 1 & Succinate & $\begin{array}{l}\text { Lysate } \\
\text { Ghosts } \\
\text { Supernatant }\end{array}$ & $\begin{array}{l}15 \cdot 5 \\
85 \cdot 2 \\
10 \cdot 7\end{array}$ & $\begin{array}{r}9 \cdot 9 \\
9 \cdot 6 \\
10 \cdot 7\end{array}$ & $\begin{array}{l}\overline{36 \cdot 3} \\
58 \cdot 1\end{array}$ & $\begin{array}{l}\overline{15 \cdot 6} \\
91 \cdot 0\end{array}$ \\
\hline 2 & Succinate & $\begin{array}{l}\text { Lysate } \\
\text { Ghosts } \\
\text { Supernatant }\end{array}$ & $\begin{array}{r}17 \cdot 3 \\
46 \cdot 5 \\
9 \cdot 9\end{array}$ & $\begin{array}{l}12 \cdot 7 \\
13 \cdot 5 \\
12 \cdot 8\end{array}$ & $\begin{array}{l}\overline{45 \cdot 6} \\
48 \cdot 0\end{array}$ & $\begin{array}{c}\overline{16 \cdot 1} \\
85 \cdot 9\end{array}$ \\
\hline & Malate & $\begin{array}{l}\text { Lysate } \\
\text { Ghosts } \\
\text { Supernatant }\end{array}$ & $\begin{array}{r}56 \cdot 6 \\
235 \cdot 2 \\
45 \cdot 6\end{array}$ & $\begin{array}{r}31 \cdot 6 \\
120 \cdot 0 \\
39 \cdot 3\end{array}$ & $\begin{array}{l}\overline{70.5} \\
66.7\end{array}$ & $\begin{array}{r}\overline{57 \cdot 0} \\
105 \cdot 5\end{array}$ \\
\hline
\end{tabular}

With both substrates, there appeared to be no decrease in the oxidative activity in the supernatant fraction from streptomycin-inhibited organism. The decreased activity in the total lysate from such organisms can be ascribed almost entirely to decreased activity in the 'ghost' fraction. When the 'ghost' fraction from normal $B$. megaterium was mixed with supernatant fraction from streptomycin-inhibited organisms, and vice versa, the resulting oxidative activities on succinate were very close to the sum of the activities of each fraction separately (Table 4).

\section{Table 4. Succinate-oxidizing activity of mixed fractions from normal and streptomycin-inhibited Bacillus megaterium $K M$}

Conditions of growth as in Table $2 ; \mathbf{C}=$ control organisms, $\mathbf{S}=$ streptomycininhibited organisms. Each 'ghost' fraction was resuspended in the same volume as that of the total lysate from which it was prepared. Figures in parentheses are activities to be expected if the activity of each fraction is additive.

\begin{tabular}{|c|c|c|c|}
\hline & None & Super & from \\
\hline & Activities & $\stackrel{\mathrm{C}}{\mathrm{C}}$ & $\underset{\text { tion/hr. }}{S}$ \\
\hline None & - & 71 & 58 \\
\hline 'Ghosts' $\int^{\mathrm{C}}$ & 103 & $\begin{array}{c}190 \\
(174)\end{array}$ & $\begin{array}{c}206 \\
(161)\end{array}$ \\
\hline from & 24 & $\begin{array}{c}75 \\
(95)\end{array}$ & $\begin{array}{c}97 \\
(82)\end{array}$ \\
\hline
\end{tabular}




\section{DISCUSSION}

The results described here would appear to exclude the occurrence of any general breakdown of the permeability barrier of Bacillus megaterium KM or Escherichia coli $\mathbf{B}$ during the period in which inhibition of growth by streptomycin becomes complete. With $E$. coli there were no large changes in the total intracellular pools of amino acids or of $260 \mathrm{~m} \mu$-absorbing compounds during this period. Moreover, protoplasts were prepared in normal yield from both these organisms when the inhibition of growth was complete; such protoplasts had a stability and a mean internal osmotic pressure which differed only slightly if at all from those of protoplasts from normal organisms. Even when streptomycin-inhibited organisms showed $Q_{\mathrm{O}_{2}}$ values which were $30 \%$ or less of those of control organisms, they could be converted to stable protoplasts, and were indeed used routinely to prepare protoplasts and total lysates. These results do not, however, exclude the possibility of changes in permeability to specific intracellular compounds or groups of compounds, such as the excretion of certain mononucleotides in $E$. coli W (Anand \& Davis, 1960), or of general changes in permeability too small to observe by the methods used here, but which might be detectable by investigating compounds released into the growth medium as was done by Anand \& Davis (1960).

A decrease in oxidative activities accompanies the inhibition of growth by streptomycin in Escherichia coli B and Bacillus megaterium KM, as well as in two strains of Staphylococcus aureus (Jackson, 1958; Hancock, 1960a) and in Bacillus pumilus (J. W. Lightbown, personal communication). With the techniques used here, conclusions as to whether this decrease precedes or follows the decrease in growth rate should be made with reservations, but the two changes are certainly closely parallel; more precise investigation of the relationship might perhaps be obtained by continuously recording the oxygen consumption of a culture. There would appear to be no a priori reason for a decreased oxidative activity which occurs as a nonspecific accompaniment of a decreased growth rate; no such change occurred in $S$. aureus after inhibition of growth by penicillin (Gale \& Taylor, 1947) or other antibiotics (Hancock, 1960a).

Since protoplast lysates from streptomycin-inhibited Bacillus megaterium showed decreased oxidative activities relative to preparations from normal organisms, it would not appear that permeability differences are responsible for the difference in activity of the corresponding intact organisms. As differences in activity were found even in the separated 'ghost' fraction, it seems unlikely that the absence of diffusible cofactors, or the presence of diffusible inhibitors, is responsible for the decreased activity of lysates from streptomycin-inhibited organisms; the observed additive activities of mixed 'ghosts' and supernatant fractions (Table 4) would also support this conclusion. With the systems which oxidize succinate and malate, the decreased activity of total lysates appears to be due predominantly to decreased activity of the 'ghost' fraction; the activity of the supernatant fraction was not affected. The succinate-oxidizing activity in the 'ghost' fraction also differs from that in the supernatant fraction in that it is stimulated about ten-fold by phenazine methosulphate under the conditions used (Kearney \& Singer, 1956) for assaying succinic dehydrogenase, whereas the activity in the supernatant fraction shows no stimulation. It is known that the 'ghost' fraction contains virtually all of the cytochrome system 
of $B$. megaterium KM (Weibull, 1953). Decreased activity of multi-enzyme systems, such as those which oxidize succinate and malate, could clearly be caused either by alterations in the activity of enzymes per se, or by structural changes in the 'ghosts'. With $B$. megaterium there is as yet no other evidence for such a structural change, and no apparent differences can be seen by phase-contrast microscopy between the ghosts from normal and streptomycin-inhibited organisms.

I am grateful to Professor B. D. Davis and Dr J. W. Lightbown for valuable comments on this paper, and to Mr F. McManus for technical assistance.

\section{REFERENCES}

Anand, N. \& Davis, B. D. (1960). Damage by streptomycin to the cell membrane of Escherichia coli. Nature, Lond. 185, 22.

Brenner, S., Dark, F. A., Gerhardt, P., Jeynes, M. H., Kandler, O., Kellenberger, E., Kuieneberger-Nobel, E., McQuillen, K., Rubio-Huertos, M., Salton, M. R. J., Strange, R. E., Tomcsik, J. \& WeIBuld, C. (1958). Bacterial protoplasts. Nature, Lond. $181,1713$.

Britten, R. J. (1956). Effect of the osmotic strength of the growth medium on the amino acid pool of Escherichia coli. Science, 124, 935.

Gale, E. F. \& TAYLOR, E. S. (1947). The assimilation of amino acids by bacteria. 5. The action of penicillin in preventing the assimilation of glutamic acid by Staphylococcus aureus. J. gen. Microbiol. 1, 314.

Hancock, R. $(1960 a)$. The bactericidal action of streptomycin on Staphylococcus aureus and some accompanying biochemical changes. J. gen. Microbiol. 23, 179.

HANCOCK, R. $(1960 b)$. Reduction in oxidative activities of some bacteria during inhibition of growth by streptomycin. Biochem. J. 76, $69 \mathrm{P}$.

JACKSON, F. L. (1958). Streptomycin and bacterial respiration. Nature, Lond. 181, 281.

Kearney, E. B. \& Singer, T. P. (1956). Studies on succinic dehydrogenase. I. Preparation and assay of the soluble dehydrogenase. J. biol. Chem. $219,963$.

Lowry, O. H., Rosebrough, N. J., Farr, A. L. \& Randall, R. J. (1951). Protein measurement with the Folin phenol reagent. J. biol. Chem. 193, 265.

MAHLER, H. P. \& Fraser, D. (1956). Reproduction of bacteriophage $T_{3}$ in protoplasts of Escherichia coli strain B. Biochim. biophys. Acta, 22, 197.

McQuillen, K. \& Roberts, R. B. (1954). The utilisation of acetate for synthesis in Escherichia coli. J. biol. Chem. 207, 81.

Mitchell, P. \& Moyle, J. (1956a). Liberation and osmotic properties of the protoplasts of Micrococcus lysodeikticus and Sarcina lutea. J. gen. Microbiol. 15, 512.

Mrtchell, P. \& Moyle, J. (1956b). Osmotic function and structure in bacteria. Symp. Soc. gen. Microbiol. 6, 150.

MrTCHELl, P. \& MOYLE, J. (1957). Autolytic release and osmotic properties of 'protoplasts' from Staphylococcus aureus. J. gen. Microbiol. 16, 184.

Storck, R. \& Wachsman, J. T. (1957). Enzyme localisation in Bacillus megaterium. J. Bact. 73, 784.

WeIBULL, C. (1953). Characterisation of the protoplasmic constituents of Bacillus megaterium. J. Bact. 66, 696.

Weibull, C., Beckman, H. \& Bergström, L. (1959). Localisation of enzymes in Bacillus megaterium strain M. J. gen. Microbiol. 20, 519. 